\title{
Use of the vaccination register to evaluate influenza vaccine coverage in seniors in the 2010/11 influenza season, Navarre, Spain
}

I Aguilar1, M Reyes ${ }^{1}$, I Martínez-Baz',2, M Guevara ${ }^{1,2}$, E Albéniz³, M J Belza ${ }^{2,4}$, J Castilla (jcastilc@navarra.es) ${ }^{1,2}$

1. Instituto de Salud Pública de Navarra (Public Health Institute of Navarre), Pamplona, Spain

2. CIBER Epidemiología y Salud Pública (Biomedical Research Network of Epidemiology and Public Health), Spain

3. Dirección de Atención Primaria (Primary Health Care Department), Pamplona, Spain

4. Escuela Nacional de Sanidad (National School of Health), Instituto de Salud Carlos III, Madrid, Spain

Citation style for this article:

Aguilar I, Reyes M, Martínez-Baz I, Guevara M, Albéniz E, Belza MJ, Castilla J. Use of the vaccination register to evaluate influenza vaccine coverage in seniors in the 2010/11 influenza season, Navarre, Spain . Euro Surveill. 2012;17(17):pii=20154. Available online: http://www.eurosurveillance.org/ViewArticle.

aspx?Articleld=20154

Article submitted on 03 October 2011/ published on 26 April 2012

People aged 65 and older have a high risk of suffering from complications of influenza, therefore it is recommended that they receive annual influenza vaccination. However, vaccination coverage falls far short of the target of $75 \%$. In this study we use the vaccination register to evaluate the coverage of influenza vaccine in non-institutionalised persons aged 65 and over in Navarre, Spain, in the $2010 / 11$ season $(104,427$ persons). Vaccination coverage was $58.6 \%$, lower than the $62.7 \%$ coverage in the $2009 / 10$ season. In the multivariate analysis, lower coverage was associated with being female, age under 80 or over 94 years, immigrant status and hospitalisation in the previous year. In contrast, persons with major chronic conditions, high level of dependence or those with more visits to the general practitioner in the previous year had higher vaccination coverage. Influenza vaccination in the previous season was a strong predictor of vaccination in the current season (odds ratio: 37.0 , 95\% confidence interval: $35.7-38.4)$. The vaccination register has been shown to be useful to monitor the coverage of influenza vaccination in seniors and may help guide strategies to improve coverage.

\section{Introduction}

Computerised vaccination records have been shown to be useful in reducing the frequency of missed opportunities for immunisation, facilitating reminder notices, preventing over-immunisation, and providing information about immunisation delivery and documentation on a population level $[1,2]$. Vaccination records can also be useful tools for clinicians and public health officials, for monitoring immunisation coverage and for providing feedback to clinicians, patients and case management workers [3,4].

Seasonal influenza can cause serious complications in the elderly and in subjects with chronic health conditions. Annual vaccination against influenza is the most effective measure for reducing morbidity and mortality associated with this disease [5-7]. In Spain, the recommendations for preventing seasonal influenza include annual vaccination of all people aged 65 and over, but the coverage reached in this population is far from the $75 \%$ target proposed by the World Health Assembly for 2010 [8]. This highlights the importance of monitoring the vaccination coverage and identifying possible ways to improve it.

The present study aims to explore the utility of a population-based vaccination register to quantify seasonal influenza vaccine coverage and to describe its determinants in non-institutionalised subjects aged 65 and older.

\section{Methods}

\section{Vaccination register}

The Regional Health Service of Navarre, Spain, provides free healthcare to approximately $97 \%$ of the population of the region (642,051 inhabitants). All Navarre residents at birth are registered into the healthcare computerised system and people who change their residence to Navarre are registered at the first visit to a healthcare centre. From 2000 to 2004 the computerised medical record was implemented throughout the whole Navarre Health Service, in both hospitals and primary care. A specific section in the computerised medical record is provided to register vaccinations. Whenever health professionals administer a dose of vaccine they must register online the type of vaccine, dose number, brand, batch, date of administration, person who administers it and possible incidents. The type and brand of vaccine are registered using predefined codes to avoid errors and make it easier to consult the information recorded. Software filters are applied to ensure valid data entry. The same registration system is used for all vaccines, both those administered in childhood 
or adult vaccination programmes and all other vaccines that are not included in these programmes. The information in this register is available to the physician and is part of each patient's computerised medical record. It provides an alert about the doses required to complete the vaccination schedules and performs the functions of the vaccination record, regardless of whether the patient retains a paper copy.

The information contained in the vaccination register is exported weekly to an individual-level database. In this format, the information from the vaccine register can be linked to other databases (as described below) with complementary data, such as data on demographics or data on incidence of vaccine-preventable diseases. The combined data can be used to monitor the coverage of each vaccine, to plan improvements in coverage, to detect vaccine failures and to evaluate the effectiveness of different vaccines.

\section{Influenza vaccination campaign}

In the $2010 / 11$ season, the influenza vaccination campaign in Navarre took place from 11 October to 26 November, following a system similar to that used in previous seasons [9]. The trivalent inactivated vaccine was used, with the composition recommended by the World Health Organization [10]. In Navarre, the vaccine was indicated for all persons aged 60 and over, and for persons with chronic diseases that increase the risk of influenza complications [9]. The vaccines for this programme were purchased centrally by the regional government and were distributed to all primary healthcare centres. Before the vaccination campaign began, an information campaign focusing on the target population was carried out by means of posters in the healthcare centres and notices in the media to let people know the population groups for whom the vaccine was indicated and where they could go to be vaccinated. Physicians and nurses in primary care centres were sent a detailed protocol of the programme with the objectives, dates, indications, logistics, instructions on how to register the doses administered, and phone numbers for further information [9]. Healthcare staff in the healthcare centres took advantage of all contacts with patients to remind them of the benefits of being vaccinated against influenza.

\section{Study population and variables}

Using computerised databases of physicians, we selected all non-institutionalised persons aged 65 and older who were covered by the Navarre Health Service at the beginning of the vaccination campaign. According to the national and regional protocols all subjects in this study population had indication for influenza vaccination [9]. From the computerised database of primary healthcare, we obtained the following baseline variables: sex, age, migrant status, district of residence, major chronic conditions, high level of dependence (Barthel's index 40 ), number of outpatient visits during the previous 12 months and hospitalisation in the previous 12 months. Residence in municipalities
TABLE 1

Characteristics of the population aged 65 and older and respective 2010/11 seasonal influenza vaccine coverage, Navarre, Spain $(n=104,427)$

\begin{tabular}{|c|c|c|}
\hline Characteristic & $\begin{array}{l}\text { Number of } \\
\text { people (\%) }\end{array}$ & $\begin{array}{l}\text { Number of } \\
\text { vaccinated } \\
\text { people (\%) }\end{array}$ \\
\hline Total & $104,427(100)$ & $61,195(58.6)$ \\
\hline \multicolumn{3}{|l|}{ Sex } \\
\hline Male & $45,712(43.8)$ & $27,201(59.5)$ \\
\hline Female & $58,715(56.2)$ & $33,994(57.9)$ \\
\hline \multicolumn{3}{|l|}{ Age group, years } \\
\hline $65-69$ & $27,543(26.4)$ & $12,605(45.8)$ \\
\hline $70-74$ & $21,995(21.1)$ & $12,715(57.8)$ \\
\hline $75-79$ & $22,353(21.4)$ & $14,289(63.9)$ \\
\hline $80-84$ & $17,220(16.5)$ & $14,289(63.9)$ \\
\hline $85-89$ & $10,291(9.9)$ & $6,905(67.1)$ \\
\hline $90-94$ & $3,749(3.6)$ & $2,426(64.7)$ \\
\hline $95-99$ & $1,081(1.0)$ & $601(55.6)$ \\
\hline$\geq 100$ & $195(0.2)$ & $61(31.3)$ \\
\hline \multicolumn{3}{|l|}{ Number of major chronic conditions } \\
\hline 0 & $45,049(43.1)$ & $22,750(50.5)$ \\
\hline 1 & $33,767(32.3)$ & $21,204(62.8)$ \\
\hline$>1$ & $25,611(24.5)$ & $17,241(67.3)$ \\
\hline \multicolumn{3}{|l|}{ Major chronic conditions } \\
\hline Liver cirrhosis & $2,214(2.1)$ & $1,320(59.6)$ \\
\hline Diabetes & $20,827(19.9)$ & $13,545(65.0)$ \\
\hline Heart disease & $21,333(20.4)$ & $14,261(66.8)$ \\
\hline Haematological cancer & $1,026(1.0)$ & $629(61.3)$ \\
\hline Non-haematological cancer & $16,806(16.1)$ & $10,539(62.7)$ \\
\hline Immunological disease & $838(0.8)$ & $525(62.6)$ \\
\hline Pulmonary disease & $15,038(14.4)$ & $10,101(67.2)$ \\
\hline Renal diseases & $8,590(8.2)$ & $5,814(67.7)$ \\
\hline Dementia & $4,429(4.2)$ & $2,926(66.1)$ \\
\hline Stroke & $6,848(6.6)$ & $4,516(65.9)$ \\
\hline Rheumatic disease & $1,800(1.7)$ & $1,117(62.1)$ \\
\hline Morbid obesity $\left(\mathrm{BMI} \geq 40 \mathrm{~kg} / \mathrm{m}^{2}\right)$ & $1,962(1.9)$ & $1,197(61.0)$ \\
\hline \multicolumn{3}{|c|}{ Visits as outpatient in the previous year } \\
\hline $0-3$ & $36,670(35.1)$ & $16,928(46.2)$ \\
\hline 4-7 & $31,403(30.1)$ & $19,415(61.8)$ \\
\hline 17 & $36,354(34.8)$ & $24,852(68.4)$ \\
\hline \multicolumn{3}{|l|}{ Seasonal influenza vaccine } \\
\hline $2009 / 10$ & $64,245(61.5)$ & $55,700(86.7)$ \\
\hline $2008 / 09$ & $62,385(59.7)$ & $53,604(85.9)$ \\
\hline $2007 / 08$ & $58,607(56.1)$ & $50,332(85.9)$ \\
\hline $\begin{array}{l}\text { Vaccinated in all three previous } \\
\text { seasons }\end{array}$ & $49,247(47.2)$ & 44,699 (90.8) \\
\hline \multicolumn{3}{|l|}{ Place of residence } \\
\hline Rural area & $50,341(48.2)$ & $30,010(59.6)$ \\
\hline Urban area & $54,086(51.8)$ & $31,185(57.7)$ \\
\hline Immigrant & $1,699(1.6)$ & $542(31.9)$ \\
\hline High level of dependence & $858(0.8)$ & $621(72.4)$ \\
\hline $\begin{array}{l}\text { Hospitalisation in the previous } \\
\text { year }\end{array}$ & $12,191(11.7)$ & $7,772(63.8)$ \\
\hline
\end{tabular}

BMI: Body mass index. 
with more than 10,000 inhabitants was considered urban and any other was defined as rural. The major chronic conditions were defined according to the International Classification of Primary Care, version 2 [11], and included: heart disease (K71, K74-77, K81-K84, K99), lung disease (R79, R95, R96, R99), renal disease (U99), cancer (A79, B72-B74, D74-D78, F74, H75, K72, L71, N74, N76, R84, R85, S77, S79, T71, T73, U75-U77, $\mathrm{U}_{79}, \mathrm{~W}_{72}-\mathrm{W}_{73}, \mathrm{X}_{75}-\mathrm{X}_{77}, \mathrm{X}_{81}, \mathrm{Y}_{77}-\mathrm{Y}_{79}$ ), diabetes (T89, T9o), liver cirrhosis (D97), dementia (P70), stroke (K90, K91), immunodeficiency or transplants (B78, B79, B90, D28, K28, U28), rheumatic disease (L88) and morbid obesity (body mass index of $40 \mathrm{~kg} / \mathrm{m}^{2}$ or greater).

From the vaccination register we obtained information on influenza vaccination in the 2010/11 season, as well as influenza vaccination in the three previous seasons. Individuals with no information on vaccination status were considered unvaccinated.

The Navarre Ethical Committee for Medical Research approved the study protocol.

\section{Analysis}

This study was conducted in Navarre after the 2010/11 influenza vaccination campaign was concluded. The distribution of the baseline covariates in the population was evaluated. Age was categorised in 5-year groups, and number of outpatient visits in the previous year was categorised in tertiles.

Influenza vaccine coverage in the $2010 / 11$ season was quantified as the percentage of people vaccinated in each population group. This data was compared with coverage in the previous seasons which was calculated the same way as for 2010/11. Possible determinants of influenza vaccine coverage in the 2010/11 season were investigated using univariate and multivariate logistic regression models. The analyses were conducted using SPSS 18.

\section{Results}

Characteristics of the population

A total of 104,427 non-institutionalised individuals aged 65 or older were included in the analysis. They represented $94.4 \%$ of the population in this age group in the region. The $5.6 \%$ remaining were those who were institutionalised or people without coverage by the public healthcare service of Navarre. Some 32,536 ( $31 \%)$ were aged 80 or older, 58,715 (56\%) were women, and 59,378 (57\%) presented at least one major chronic condition, most frequently, heart disease $(21,333,20 \%)$

\section{TABLE 2}

Determinants of 2010/11 seasonal influenza vaccine coverage, Navarre, Spain $(n=104,427)$

\begin{tabular}{|c|c|c|c|}
\hline Factor & $\begin{array}{c}\text { Crude odds ratios } \\
\text { (95\% confidence interval) }\end{array}$ & $\begin{array}{l}\text { Adjusted odds ratios } \\
(95 \% \text { confidence interval })^{\mathrm{a}}\end{array}$ & $\mathrm{P}$ \\
\hline Female vs. male & $0.94(0.91-0.96)$ & $0.91(0.88-0.93)$ & $<0.001$ \\
\hline \multicolumn{4}{|l|}{ Age group (in years) } \\
\hline $65-69$ & Reference & Reference & \\
\hline $70-74$ & $1.62(1.57-1.68)$ & $1.56(1.50-1.62)$ & $<0.001$ \\
\hline $75-79$ & $2.10(2.03-2.18)$ & $1.91(1.84-1.98)$ & $<0.001$ \\
\hline $80-84$ & $2.44(2.35-2.54)$ & $2.18(2.09-2.27)$ & $<0.001$ \\
\hline $85-89$ & $2.42(2.31-2.53)$ & $2.17(2.07-2.28)$ & $<0.001$ \\
\hline $90-94$ & $2.17(2.02-2.33)$ & $2.03(1.89-2.19)$ & $<0.001$ \\
\hline $95-99$ & $1.48(1.31-1.68)$ & $1.50(1.32-1.70)$ & $<0.001$ \\
\hline$\geq 100$ & $0.54(0.40-0.73)$ & $0.66(0.48-0.90)$ & 0.008 \\
\hline \multicolumn{4}{|l|}{ Major chronic conditions } \\
\hline None & Reference & Reference & \\
\hline 1 & $1.65(1.61-1.70)$ & $1.41(1.36-1.45)$ & $<0.001$ \\
\hline$>1$ & $2.02(1.96-2.09)$ & $1.50(1.45-1.55)$ & $<0.001$ \\
\hline \multicolumn{4}{|l|}{ Visits as outpatient in the previous year } \\
\hline $0-3$ & Reference & Reference & \\
\hline $4-7$ & $1.89(1.83-1.95)$ & $1.79(1.74-1.85)$ & $<0.001$ \\
\hline 17 & $2.52(2.45-2.60)$ & $2.19(2.12-2.27)$ & $<0.001$ \\
\hline Immigrant vs. Spanish citizen & $0.33(0.29-0.36)$ & $0.40(0.36-0.45)$ & $<0.001$ \\
\hline Residence in urban area vs. rural area & $0.92(0.90-0.95)$ & $1.00(0.98-1.03)$ & 0.836 \\
\hline High level of dependence & $1.86(1.60-2.16)$ & $1.27(1.09-1.49)$ & 0.003 \\
\hline Hospitalisation in the previous year & $1.28(1.23-1.33)$ & $0.89(0.85-0.93)$ & $<0.001$ \\
\hline
\end{tabular}

${ }^{a}$ Logistic regression model including all the variables listed in the table. 
or diabetes $(20,827,20 \%)$, and $12,191(12 \%)$ had been hospitalised in the previous year (Table 1).

\section{Influenza vaccine coverage}

in the 2010/11 season

Overall influenza vaccine coverage reached $58.6 \%$ of the study population. The coverage was slightly higher in men (59.5\%) and differed by age group, with the highest vaccine coverage observed for the 85 to 89 year age group $(67.1 \%)$ and the lowest for subjects older than 99 years $(31.3 \%)$ or aged 65 to 69 years $(45.8 \%)$.

Subjects with major chronic conditions were more likely to be vaccinated, and an upward trend was seen with increasing number of diseases. People with renal $(67.7 \%)$, pulmonary $(67.2 \%)$ and heart diseases $(66.8 \%)$ had the highest vaccination rates.

The proportion of vaccinated people increased with the number of visits as outpatient in the previous year,

\section{FIGURE}

Major chronic conditions as determinants of 2010/11 seasonal influenza vaccine coverage, Navarre, Spain $(\mathrm{n}=104,427)$

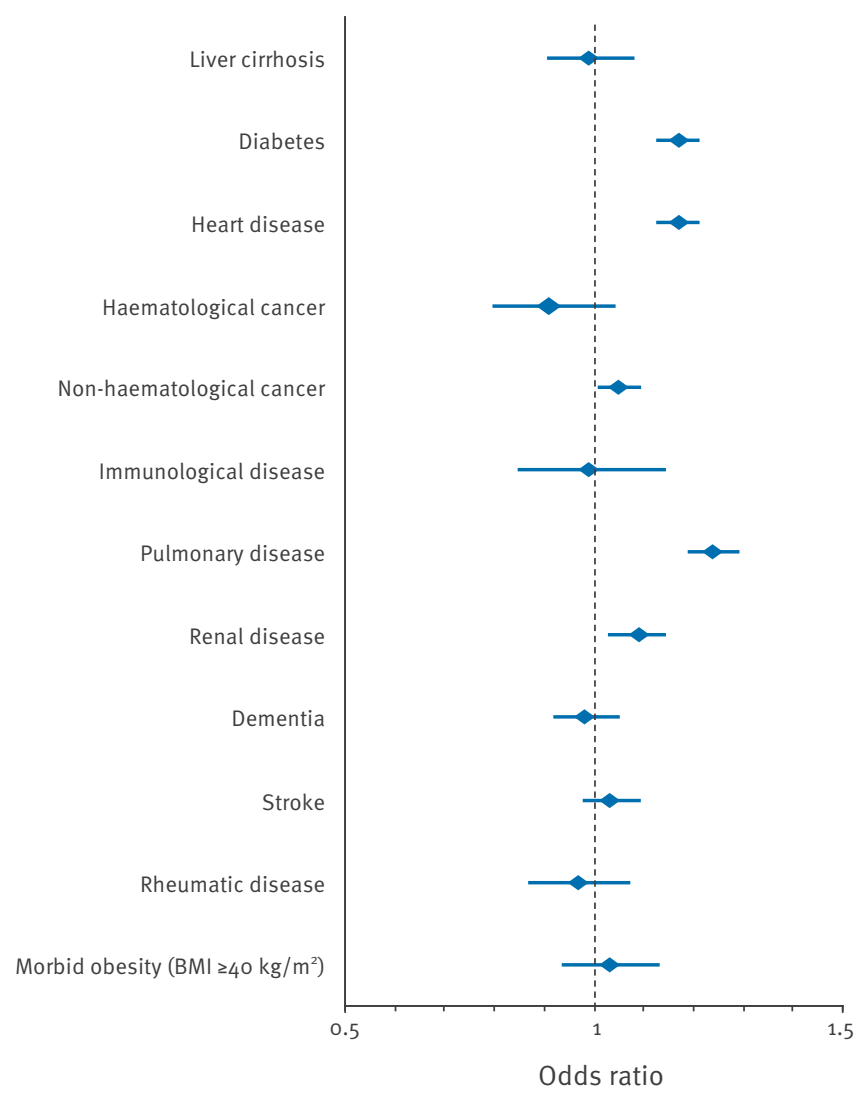

BMI: Body Mass Index.

Odds ratios obtained from a logistic regression analysis adjusted for sex, age, outpatient visits in the previous 12 months, immigrant status, urban/rural residence, level of dependence and hospitalisation in the previous 12 months. from $46.2 \%$ in subjects with zero to three visits to $68.4 \%$ in those with more than seven visits.

The highest influenza vaccine coverage was observed in subjects who had been vaccinated in all three previous seasons ( $90.8 \%)$. Vaccination coverage was also high in subjects who had been vaccinated against influenza in the $2009 / 10$ season ( $86.7 \%$ ).

Similar coverage was observed among persons with urban or rural residence. Immigrants had the lowest vaccination rates (31.9\%) (Table 1 ).

\section{Determinants of seasonal}

influenza vaccine coverage

Multivariate analyses showed that having at least one major chronic condition, more than three visits as an outpatient in the previous year, and high level of dependence were associated with greater vaccination uptake. Age was significantly associated with vaccine uptake, especially in the age group 85 to 89 years (odds ratio (OR): $2.18,95 \%$ confidence interval $(\mathrm{Cl})$ : 2.09-2.27) and 80 to 84 years (OR: $2.17,95 \% \mathrm{Cl}: 2.07-$ 2.28) as compared with those aged 65 to 69 . On the other hand, being female, being older than 99 years of age, being an immigrant or having been hospitalised in the previous year were associated with a lower seasonal vaccination uptake. Residence in an urban area was not significantly associated with differences in seasonal influenza vaccine coverage (Table 2).

When each type of major chronic condition was considered separately after adjusting for all other covariates, vaccination rates were found to be higher among people with pulmonary disease (OR: $1.25,95 \% \mathrm{Cl}: 1.20-$ 1.30), diabetes and heart disease (OR: $1.18,95 \% \mathrm{Cl}$ : 1.14-1.22 for both diseases). Non-haematological cancer and renal disease were also associated with significantly higher influenza vaccine coverage. The other chronic conditions evaluated were not associated with vaccination uptake (Figure).

When influenza vaccination in the previous season was introduced in the adjusted model, it was found to be a strong predictor of vaccination in the current season (OR: $37.0,95 \% \mathrm{Cl}: 35.7-38.4$ ). Having been vaccinated in all three previous seasons improved the predictive value (OR: $55.8,95 \%$ Cl: $53.5-58.2$ ) (Table 3).

\section{Time trends in vaccine coverage}

The vaccine register made it possible to obtain estimates of influenza vaccination coverage in seniors in the most recent seasons using comparable criteria. In 2006 influenza vaccine coverage in non-institutionalised persons aged 65 or over was $59.9 \%$, rising to $61.3 \%$ in 2007 and peaking at $62.7 \%$ in the $2008 / 09$ and $2009 / 10$ seasons. The coverage achieved in the 2010 campaign (58.6\%) represents a decline with respect to coverage in the previous campaign ( $p<0.001$ ) and breaks the previous upward trend. 


\section{Discussion}

Our results show the utility of the vaccination register in monitoring the coverage of influenza vaccination in seniors in Navarre and in identifying factors that affect this coverage.

Vaccination registries have been shown to be useful in clinical assessment and in estimating coverage [12]. In Navarre, the vaccine register is used primarily so that physicians and nurses can consult the vaccine status of their patients at any time and to programme the dates to administer the doses of the vaccine schedule. This register allows more rigorous research, because it contains individual-level information that can be used to conduct population-based studies to measure the impact of vaccination programmes, to evaluate vaccine effectiveness, and to monitor vaccine safety [13-21].

Although the completeness and accuracy of the vaccine register have not been evaluated in Navarre, there are multiple data that support it. The number of doses registered is very close to the number of doses distributed from the regional vaccination programme, and the small differences can be explained on the basis of stock, expired doses, cold chain failure or other incidents. No discrepancies have been detected in healthcare or public health practice between the vaccination data in the register and the information in patients' vaccination records. The person who administers the vaccine documents it at the same time. Furthermore, one of the indicators used in quality assurance of primary care in Navarre is the vaccination coverage in people for whom the vaccine was indicated, which serves as an incentive for physicians to record all doses administered.

Vaccination registries make it possible to evaluate coverage continually in the whole population in a way that is comparable over time. Vaccination coverage has also been studied through surveys in population samples [22-24]. Although the coverage of influenza vaccination in our study $(58.6 \%)$ is within the range of what other authors have reported [22-24], it is far from the programme objective and leaves considerable margin for improvement [8]. Vaccination coverage in Navarre had increased in recent years through efforts to improve information and vaccine uptake. However, coverage fell appreciably after the influenza $A\left(\mathrm{H}_{1} \mathrm{~N}_{1}\right)$ pdmog pandemic in 2009. Women had lower vaccination coverage than men, and people with chronic conditions were more likely to be immunised, in particular those with pulmonary disease, diabetes or heart disease. All these results are consistent with what has been described in the literature $[22,25]$. However, coverage in persons with major chronic conditions could still be improved. Hospitalisation in the previous year was associated with a lower probability of vaccination, which suggests that in these patients' primary care, measures like vaccinations may be forgotten, despite the fact that their potential benefit in these persons may be even greater.

Influenza vaccination in previous seasons was a strong predictor of vaccination in the current season, which shows that efforts to improve vaccine uptake may be rewarded by increased commitment to the programme in successive seasons. It is likely that people's objections to and reservations about the vaccine are largely overcome after being vaccinated for the first time.

Although influenza vaccination is considered an effective measure against influenza, the decision whether or not to accept the vaccine is influenced by many factors. Low immunisation coverage is usually attributed to a combination of patient characteristics, beliefs and attitudes about vaccines, and barriers to access [22]. In this study we only evaluated factors depending on the subject, even though some healthcare factors may also influence the vaccination coverage achieved. Some authors have pointed out that the single most important factor is the recommendation of a healthcare

TABLE 3

Previous seasonal influenza vaccinations as determinants of 2010/11 seasonal influenza vaccine uptake, Navarre, Spain $(\mathrm{n}=104,427)$

\begin{tabular}{|c|c|c|c|}
\hline Vaccination status & $\begin{array}{c}\text { Crude odds ratios } \\
\text { (95\% confidence interval) }\end{array}$ & $\begin{array}{l}\text { Adjusted odds ratios ( } 95 \% \\
\text { confidence interval) }\end{array}$ & $P$ \\
\hline \multicolumn{4}{|l|}{ Analysis 1} \\
\hline Not vaccinated in $2009 / 10$ season & Reference & Reference & - \\
\hline Vaccinated in $2009 / 10$ season & $41.2(39.7-42.7)$ & $37.0(35.7-38.4)$ & $<0.001$ \\
\hline \multicolumn{4}{|l|}{ Analysis 2} \\
\hline Not vaccinated in $2009 / 10$ season & Reference & Reference & - \\
\hline Vaccinated in $2009 / 10$ but not in all three previous seasons & $17.4(16.6-18.2)$ & $16.2(15.5-17.0)$ & $<0.001$ \\
\hline Vaccinated in $2009 / 10$ and in all three previous seasons & $62.0(59.5-64.7)$ & $55.8(53.5-58.2)$ & $<0.001$ \\
\hline
\end{tabular}

a Logistic regression model adjusted for sex, age, major chronic conditions, outpatient visits in the previous 12 months, immigrant status, urban/rural residence, level of dependence and hospitalisation in the previous 12 months. 
professional [24]. When vaccination in elderly people has been explored, perceptions of enjoying good health and the feeling of not being susceptible to influenza were the reasons most frequently mentioned for non-compliance. It was also associated with the opinion that the complications of influenza were not dangerous [25].

Our results demonstrate the need to improve influenza vaccination coverage in Navarre. Activities should be directed to increasing awareness of those who belong to high-risk groups, encouraging healthcare workers to proactively propose influenza vaccination, promoting the use of patient reminders, and developing effective communication and education campaigns $[6,26]$.

\section{Conclusion}

The Navarre vaccine register has been shown to be a useful tool in determining vaccine coverage in the population, its evolution over time, and the influence of different factors. This information has made it possible to detect possible ways to improve coverage and may aid the design of more efficient measures to achieve this objective.

\section{Acknowledgments}

This work was supported by the I-MOVE (Influenza Monitoring Vaccine Effectiveness in Europe) programme funded by the European Centre for Disease Prevention and Control (ECDC), and by the Carlos III Institute of Health (GRog/0028 and PSo9/01179).

\section{References}

1. Kempe A, Beaty BL, Steiner JF, Pearson KA, Lowery NE, Daley $M F$, et al. The regional immunization registry as a public health tool for improving clinical practice and guiding immunization delivery policy. Am J Public Health. 2004;94(6):967-72.

2. Centers for Disease Control Prevention (CDC). Progress in Development of Immunization Registries --United States, 2000. MMWR Morb Mortal Wkly Rep. 2001;50(1):3-7.

3. Development of community- and state-based immunization registries. CDC response to a report from the National Vaccine Advisory Committee. MMWR Recomm Rep. 2001;50(RR-17):1-17.

4. Davidson AJ, Melinkovich P, Beatty BL, Chandramouli V, Hambidge SJ, Phibbs SL, et al. Immunization registry accuracy. Improvement with progressive clinical application. Am J Prev Med. 2003;24(3):276-80.

5. Nichol KL, Nordin JD, Nelson DB, Mullooly JP, Hak E. Effectiveness of influenza vaccine in the community-dwelling elderly. N Engl J Med. 2007;357(14):1373-81.

6. Blank PR, Schwenkglenks M, Szucs TD. Influenza vaccination coverage rates in five European countries during season $2006 / 2007$ and trends over six consecutive seasons. BMC Public Health. 2008;8:272.

7. Rodríguez MI, Gatón del Amo M, Robles V, Rubio-Domínguez J. Factores determinantes de la vacunación antigripal en mayores de 65 años [Factors determining flu vaccination in the over 65s]. Aten Primaria. 2006;37(7):381-5. Spanish.

8. World Health Organization (WHO). Fifty-Sixth World Health Assembly. Prevention and control of influenza pandemics and annual epidemics. Geneva:WHO; 28 May 2003. Available from: http://apps.who.int/gb/archive/pdf_files/WHA56/ea56r19.pdf

9. Instituto de Salud Pública de Navarra. Protocolo de vacunación antigripal 2010-2011 [Influenza vaccination protocol 20102011]. Boletín Informativo $n$ 우 59. Septiembre 2010. Spanish. Available from: http://www.navarra.es/NR/rdonlyres/ AECCD760-AB2A-4841-818A-FA53478FD6DC/165169/ BOL59INTCampanaGripe.pdf
10. Recommended viruses for influenza vaccines for use in the 2010-2011 northern hemisphere influenza season. Wkly Epidemiol Rec. 2010;85:81-92.

11. ICPC-2. International Classification of Primary Care, 2nd edn. Oxford: Oxford University Press; 1998.

12. Zimmerman LA, Bartlett DL, Enger KS, Gosney K, Williams WG Influenza vaccination coverage: findings from immunization information systems. BMC Pediatr. 2007;7:28.

13. Placzek H, Madoff LC. The use of immunization registrybased data in vaccine effectiveness studies. Vaccine. 2011;29(3):399-411.

14. Castilla J, Morán J, Martínez-Artola V, Reina G, Martínez-Baz I, García Cenoz M, et al. Effectiveness of trivalent seasonal and monovalent influenza $\mathrm{A}\left(\mathrm{H}_{1} \mathrm{~N}_{1}\right) 2009$ vaccines in population with major chronic conditions of Navarre, Spain: 2010/11 mid-season analysis. Euro Surveill. 2011;16(7):pii=19799. Available from: http://www.eurosurveillance.org/ViewArticle. aspx?Articleld=19799

15. Barricarte A, Castilla J, Gil-Setas A, Torroba L, Navarro-Alonso JA, Irisarri F, et al. Effectiveness of the 7-valent pneumococcal conjugate vaccine: a population-based case-control study. Clin Infect Dis. 2007;44(11):1436-41.

16. Castilla J, García Cenoz M, Arriazu M, Fernández Alonso M, Martínez Artola V, Etxeberria J, et al. Effectiveness of the Jeryl Lynn-containing vaccine in Spanish children. Vaccine. 2009;27(15): 2089-93.

17. Guevara M, Barricarte A, Gil Setas A, García Irure JJ, Beristain $X$, Torroba L, et al. Changing epidemiology of invasive pneumococcal disease following increased coverage of the heptavalent conjugate vaccine in Navarre, Spain. Clin Microbiol Infect. 2009;15(11):1013-9.

18. Castilla J, Morán J, Martínez-Artola V, Fernández-Alonso M, Guevara M, García Cenoz M, et al. Effectiveness of the monovalent influenza $A\left(\mathrm{H}_{1} \mathrm{~N}_{1}\right) 2009$ vaccine in Navarre, Spain, 2009-2010: cohort and case-control study. Vaccine. 2011;29(35):5919-24.

19. Castilla J, Beristain X, Martínez-Artola V, Navascués A, García Cenoz M, Alvarez N, et al. Effectiveness of rotavirus vaccines in preventing cases and hospitalizations due to rotavirus gastroenteritis in Navarre, Spain. Vaccine. 2012;30(3):539-43.

20. Castilla J, Martínez-Artola V, Salcedo E, Martínez-Baz I, García Cenoz M, Guevara M, et al. Vaccine effectiveness in preventing influenza hospitalizations in Navarre, Spain, 2010-2011: Cohort and case-control study. Vaccine. 2012;30(2):195-200.

21. Rodríguez-Rieiro C, Esteban-Vasallo MD, Domínguez-Berjón MF, Astray-Mochales J, Iniesta-Fornies D, Barranco-Ordoñez $D$, et al. Coverage and predictors of vaccination against 2009 pandemic $\mathrm{H}_{1} \mathrm{~N}_{1}$ influenza in Madrid, Spain. Vaccine. 2011;29(6):1332-8.

22. Sarriá Santamera A, Timoner J. Influenza vaccination in old adults in Spain. Eur J Public Health. 2003;13(2):133-7.

23. Evans MR, Watson PA. Why do older people not get immunised against influenza? A community survey. Vaccine. 2003;21(19-20):2421-7.

24. Blank PR, Schwenkglenks M, Szucs TD. Vaccination coverage rates in eleven European countries during two consecutive influenza seasons. J Infect. 2009;58(6):446-58.

25. Van Essen GA, Kuyvenhoven MM, de Melker RA. Why do healthy elderly people fail to comply with influenza vaccination? Age Ageing. 1997;26(4):275-9.

26. Kroneman M, van Essen GA, John Paget W. Influenza vaccination coverage and reasons to refrain among high-risk persons in four European countries. Vaccine. 2006;24:622-8. 